\title{
Lower-Frequency Event-Related Desynchronization: A Signature of Late Mismatch Responses to Sounds, Which Is Reduced or Absent in Children with Specific Language Impairment
}

\author{
Dorothy V. M. Bishop, Mervyn J. Hardiman, and Johanna G. Barry \\ Department of Experimental Psychology, University of Oxford, OX1 3UD Oxford, United Kingdom
}

Poor discrimination of nonlinguistic sounds has been implicated in language-learning problems in children, but research evidence has been inconsistent. This study included 32 participants with specific language impairment (SLI) and 32 typically developing controls aged 7-16 years. Frequency discrimination thresholds were estimated in a task where participants had to distinguish a higher-frequency tone from a $1000 \mathrm{~Hz}$ tone. Neurophysiological responses were assessed in an oddball paradigm. Stimuli were either 1030 or $1200 \mathrm{~Hz}$ pure tones (deviants) presented in a series of standard $1000 \mathrm{~Hz}$ tones, or syllables (deviant [da] or [bi] in a series of standard /ba/). On the behavioral task, children (7- to 11-year-olds) had high thresholds, regardless of language status, but teenagers (12-16 years) with SLI had higher thresholds than their controls. Conventional analysis of electrophysiological responses showed no difference between groups for the mismatch negativity (MMN), but the late discriminative negativity (LDN) was reduced in amplitude for smaller deviants in participants with SLI. Time-frequency analysis revealed that, whereas the MMN reflected enhanced intertrial coherence in the theta frequency band, the LDN corresponded to a period of event-related desynchronization extending across a wide low-frequency band including delta, theta, and alpha. This manifested as a drop in power in those frequencies, which was marked in the controls but reduced or absent in children with SLI across all stimulus types. This provides compelling evidence for a low-level auditory perceptual impairment in SLI that affects a processing stage after initial detection of a sound change.

\section{Introduction}

Event-related potentials (ERPs) can inform a long-standing debate in the field of developmental disorders, namely, whether language difficulties result from low-level auditory perceptual problems. Difficulties with phonological processing, i.e., the discrimination and/or categorization of speech sounds, are reliably found in both developmental dyslexia and specific language impairment (SLI) (Bishop and Snowling, 2004). There is, however, little agreement as to whether these phonological difficulties indicate domain-specific deficits in the language system, or whether they are downstream consequences of auditory impairments (Bailey and Snowling, 2003). Behavioral evidence is hard to interpret because young children who can distinguish stimuli adequately may nevertheless obtain high auditory discrimination thresholds because of poor attention, memory, or motivation.

\footnotetext{
Received April 30, 2010; revised Sept. 6, 2010; accepted Sept. 21, 2010.

The project was funded by Wellcome Trust Programme Grant 053335. We thank Kathy White, Liz Line, Ifat Yasin, and Margriet Groen for assistance with data collection. We gratefully acknowledge the help of the many schools and families who participated in the research. Without their support and enthusiasm this work would not have been possible.

This article is freely available online through the J Neurosci Open Choice option.

Correspondence should be addressed to Dorothy Bishop, Department of Experimental Psychology, University of Oxford, South Parks Road, 0xford OX1 3UD. E-mail: dorothy.bishop@psy.ox.ac.uk.

J. G. Barry's present address: MRC Institute of Hearing Research Nottingham Clinical Section, Eye, Ear Nose \& Throat Centre, Queens Medical Centre, NG7 2UH Nottingham, UK.

DOI:10.1523/JNEUROSCI.2217-10.2010

Copyright $\odot 2010$ the authors $\quad 0270-6474 / 10 / 3015578-07 \$ 15.00 / 0$
}

The mismatch negativity (MMN) has been proposed as an alternative method for assessing auditory discrimination in SLI (Cheour et al., 2000). The MMN is assessed in a paradigm where rare deviant stimuli are presented in a train of repeated standards. Responses to deviants and standards are averaged separately and then subtracted to reveal enhanced negativity to deviants, typically occurring $\sim 100-250$ ms after stimulus onset. Importantly, the MMN can be measured passively, without requiring the child to perform a task or attend to the stimuli. However, a review of MMN studies on dyslexia and SLI (Bishop, 2007) revealed wide disparities in findings. Bishop suggested that this might reflect the specific stimuli used: studies that showed significant differences between controls and language/literacy-impaired children tended to use standard-deviant pairings with small differences between stimuli. Studies also varied in the intervals used to define the MMN, with many using time windows later than the traditional 100-250 ms post-onset period. This is important because there is a later negativity in the mismatch wave, known as the late-discriminative negativity (LDN), which has different characteristics from the classic MMN (Cheour et al., 2001). Bishop et al. (2010) found that the LDN, in contrast to the MMN, was larger in children than in adults. Also, whereas the MMN becomes larger as the difference between standard and deviant increases, the opposite pattern was shown for the LDN, which was most pronounced for small deviants.

In the current study, we used time-frequency analysis as well as more conventional averaging to assess mismatch responses in early $(\mathrm{MMN})$ and late (LDN) time windows, comparing a group 
Table 1. Mean age and scores on psychometric battery in relation to age and language status

\begin{tabular}{|c|c|c|c|c|}
\hline & TD-child & TD-teen & SLI-child & SLI-teen \\
\hline Male/female ${ }^{a}$ & $10 / 6$ & $10 / 6$ & $11 / 5$ & $11 / 5$ \\
\hline Age (years) (SD) & $9.75(1.29)$ & $13.80(1.06)$ & $9.82(1.27)$ & $14.06(1.20)$ \\
\hline WASI PIQ (SD) & $102.31(9.86)$ & $101.38(12.15)$ & $98.38(9.22)$ & $100.75(9.35)$ \\
\hline Number of language tests low (SD) & $0.31(0.48)$ & $0.19(0.40)$ & $5.00(2.22)$ & $3.63(1.89)$ \\
\hline$C C C-2: G C C^{b}(S D)$ & $75.40(30.00)$ & $71.93(22.39)$ & $36.27(17.71)$ & $37.25(16.74)$ \\
\hline Test for Reception of Grammar' (SD) & $99.07(11.44)$ & $104.43(6.30)$ & $79.81(11.59)$ & $94.38(11.10)$ \\
\hline ERRNI story telling ${ }^{c}(S D)$ & $98.53(11.03)$ & $99.21(10.28)$ & $90.00(12.50)$ & $100.36(13.64)$ \\
\hline ERRNI story recall' (SD) & $101.60(13.49)$ & $100.43(10.08)$ & $86.13(18.54)$ & $101.00(15.54)$ \\
\hline ERRNI forgetting ${ }^{c}(S D)$ & $103.53(12.97)$ & $101.07(7.55)$ & $91.44(17.91)$ & $96.71(16.55)$ \\
\hline ERRNI comprehension` (SD) & $105.13(9.13)$ & $108.57(14.93)$ & $86.31(21.97)$ & $102.57(16.18)$ \\
\hline ERRNI mean length of utterance ${ }^{c}(S D)$ & $100.00(9.18)$ & $95.29(10.58)$ & $91.07(10.92)$ & $94.71(24.36)$ \\
\hline NEPSY nonword repetition ${ }^{d, e}(S D)$ & $9.67(3.60)$ & $10.86(3.70)$ & $5.44(3.54)$ & $6.31(3.57)$ \\
\hline NEPSY sentence repetition ${ }^{d}(\mathrm{SD})$ & $9.93(3.37)$ & $12.21(2.08)$ & $4.50(1.86)$ & $5.94(3.70)$ \\
\hline TOWRE sight words ${ }^{c}(S D)$ & $101.47(11.69)$ & $98.75(13.23)$ & $87.38(14.36)$ & $79.38(13.41)$ \\
\hline TOWRE phonetic decoding ${ }^{C}$ (SD) & $106.27(10.50)$ & $108.13(9.65)$ & $83.56(14.07)$ & $77.06(12.91)$ \\
\hline
\end{tabular}

Wechsler Abbreviated Scale of Intelligence (WASI) (Wechsler, 1999); the Children's Communication Checklist (cCC-2) (Bishop, 2003a); the Test for Reception of Grammar, version 2 (Bishop, 2003b); Expression, Reception and Recall of Narrative Instrument (ERRNI) (Bishop, 2004); NEPSY (Korkman et al., 1998); Test of Word Reading Efficiency (TOWRE) (Trgesen et al., 1999).

${ }^{a}$ Missing data: seven cases for $\left(C C-2\right.$, three for TROG-2, five for ERRNI, three for nonword repetition, three for sentence repetition, and one for TOWRE. ${ }^{b} \mathrm{General}$ Communication Composite (GCC): normative mean $=80$; ${ }^{c}$ scaled with mean 100, SD $15 ;{ }^{d}$ scaled with mean 10, SD $3 ;{ }^{e}$ relative to own norms.

of individuals with SLI with a control group matched by age and nonverbal ability. Both groups were subdivided into children (7-11 years) and teenagers (12-16 years), because both behavioral and electrophysiological measures of auditory processing develop over this age range. We assessed frequency-discrimination thresholds and ERPs for both tone and syllable stimuli, using large and small deviants presented in a train of standards. The hypotheses were that the group with SLI would show reductions of mismatch responses to small deviants only and that this difference would be seen for both speech and nonspeech stimuli.

\section{Materials and Methods}

Participants

Individuals with SLI $(n=32)$ were recruited from schools and classrooms for children with language or literacy problems. All obtained a scaled score of $\leq 1.3$ SD below average on at least two of 11 language measures, had a nonverbal IQ of $\geq 80$, and passed a hearing screen covering speech frequencies $(500-4000 \mathrm{~Hz})$. Two reading tests were included in the language battery, but no child was included solely on the basis of poor literacy. No child had a diagnosis of autism or was taking any psychotropic medication. Children with signs of autistic features were screened using the Social Communication Questionnaire (Rutter et al., 2003) and excluded if they scored above a cutoff score of 11 (Eaves et al., 2006).

Typically developing controls (TD group) were a subset of those participating in a study of normal development by Bishop et al. (2010). They were selected to be matched on age and nonverbal IQ to the children with SLI. All had nonverbal IQs of $\geq 80$ and passed the hearing screen. The test battery is shown in Table 1, together with the mean scores and gender distribution for the SLI and TD children.

This study was approved by the Oxford Psychiatric Research Ethics Committee, parents of all participants gave written informed consent, and the children themselves gave assent after the study was explained in age-appropriate language.

\section{Psychoacoustic assessment of frequency discrimination}

The psychoacoustic procedure used a game-like computerized task with AXB three-alternative two-interval format, with the child hearing three tones on each trial, and being asked to select the tone (first or third) that differed from the middle one. The standard stimulus was a $1000 \mathrm{~Hz}$ tone of $100 \mathrm{~ms}$ duration. The first or third tone was either another standard stimulus or a deviant stimulus of higher frequency. Frequency was adjusted adaptively depending on the child's responses to converge on a threshold corresponding to $75 \%$ correct. In general, two runs were given, unless a very low threshold ( $10 \mathrm{~Hz}$ or less) was achieved on the first run.
Thresholds (in $\mathrm{Hz}$ difference from the standard) were converted to natural logs, and the better of the two estimates was taken for analysis.

\section{Electrophysiological assessment of mismatch negativity}

Stimuli. Stimuli are described in detail by Bishop et al. (2010). Tone stimuli consisted of a standard $1000 \mathrm{~Hz}$ tone and deviants of $1200 \mathrm{~Hz}$ (large deviant) and $1030 \mathrm{~Hz}$ (small deviant). Speech stimuli consisted of the syllables [ba] (standard), [bi] (large deviant), and [da] (small deviant). Natural speech recordings were used, with intonation contours equated using Praat (Boersma and Weenink, 2005) and RMS equalization performed with GoldWave (Craig, 2008). Stimuli had durations of $175 \mathrm{~ms}$, windowed at $15 \mathrm{~ms}$, and were presented monaurally to the right ear at 86.5 dB SPL through sound-attenuating Sennheiser HD25-1 headphones.

Procedure. Standards were presented on $70 \%$ of trials, with each deviant occurring on $15 \%$ of trials in a quasi-random sequence, avoiding occurrence of two deviants in succession. Stimulus onset asynchrony was $1 \mathrm{~s}$. There were two blocks each of 333 trials, making a total of 466 standards and 100 of each deviant type. Participants were seated in a comfortable upright chair in a sound-attenuated electrically shielded booth. To help them ignore the stimuli, participants played a Gameboy or watched a silent DVD or video on a small TV screen located at a distance of $1.3 \mathrm{~m}$ away.

EEG recording and data analysis. The EEG was recorded on a SynAmps or NuAmps NeuroScan system using $\mathrm{Ag} / \mathrm{AgCl}$ sintered electrodes and a water-soluble conductive gel. Early pilot studies indicated no difference in the results obtained from the two recording systems, and the proportions of children from each group tested on each system did not differ significantly. An electrode cap was fitted to record from 28 sites, as follows: FC1, F7, FP1, FZ, FP2, F8, FC2, FT9, FC5, F3, FCZ, F4, FC6, FT10, T7, C3, CZ, C4, T8, CP5, P7, P3, PZ, P4, P8, CP6, M1, and M2. M1 or M2 was selected as reference electrode and ground was placed at AFZ. Electrooculograms were recorded from supraorbital and infraorbital electrodes on the left eye and also from electrodes placed lateral to the left and right eyes. Impedances for all electrodes were kept $<8 \mathrm{k} \Omega$. The EEG was recorded continuously on-line and stored for off-line processing. EEG data were digitized at $500 \mathrm{~Hz}$ and bandpass filtered $(0.01-70 \mathrm{~Hz}$ for SynAmps; 0.1-70 Hz for NuAmps); a $50 \mathrm{~Hz}$ notch filter was used.

Offline analysis. Data processing is described in detail by Bishop et al. (2010). This was done separately for each individual participant for tone and speech stimuli. Data were epoched into trials of $1000 \mathrm{~ms}$ duration, including $200 \mathrm{~ms}$ baseline. Re-referencing was performed to average mastoids. Trials with extreme amplitudes $( \pm 350 \mu \mathrm{v})$ were rejected to remove noisy sections of record while retaining blinks. Artifact reduction was performed using independent component analysis to identify unwanted components such as blinks, which were mathematically subtracted from the data. Further artifact rejection was then applied with a 
Table 2. Mean FD limen (log units) in relation to age and language status

\begin{tabular}{lllll}
\hline & TD-child & SLI-child & TD-teen & SLI-teen \\
\hline$N$ & 16 & 16 & $15^{a}$ & $15^{a}$ \\
FD limen (Ln Hz) (SD) & $3.78(1.34)$ & $3.32(1.63)$ & $2.01(0.88)$ & $3.15(1.24)$ \\
Mean Hz equivalent & 44.0 & 27.7 & 7.47 & 23.4 \\
\hline
\end{tabular}

Main effect of $S L I$ status: $F_{(1,58)}=1.04, p=0.311$, partial $\eta^{2}=0.018$. Main effect of age band: $F_{(1,58)}=8.52, p=$ 0.005 , partial $\eta^{2}=0.128$. Interaction, SLI status $\times$ age band: $F_{(1,58)}=5.81, p=0.019$, partial $\eta^{2}=0.091$.

${ }^{a}$ Data missing for one TD-teen and one SLI-teen because of equipment failure or scheduling problems.

cutoff of $\pm 150 \mu \mathrm{v}$. Spatial principal components were analyzed, treating channel amplitudes at each time point in the averaged waveform (all trials, standard, and deviant) as a new set of observations. Weights from the first component were then used to create a new channel consisting of the weighted average of all channels, with polarity set to be consistent with electrode FZ. Difference waveforms were created for single-trial analysis. For each stimulus type (tones or speech), this involved categorizing four types of trial— large deviants, small deviants, dummy deviants (i.e., standards preceding deviants), and all other standards. This latter set was averaged to give a mean standard response, which was then subtracted from all the other trials. We thus had three types of difference waveforms, one for each deviant and one dummy set formed by subtracting each standard-before-deviant from the average of other standards. These waveforms represent the specific activity associated with mismatch after removing the ERP common to standards and deviants. Conventional analysis of mean amplitudes for each type of difference waveform, using time windows of 100-250 ms for the MMN and 350-550 ms for the LDN, was performed. Time-frequency analysis of single trials was performed for each type of difference waveform using EEGlab software (Delorme and Makeig, 2004) to obtain two spectrally based measures. The method for frequency extraction was fast Fourier transform with Hanning window tapering, and pad ratio was set to 2 . This gave 200 estimates with subwindow centers ranging from -135.9 to $735.9 \mathrm{~ms}$, and a window size of $128 \mathrm{~ms}$. Intertrial coherence (ITC) ranged from 0 to 1 and corresponded to the extent to which individual trials had oscillations in phase at a given frequency at a given time point, i.e., the degree of temporal alignment of auditory responses. Event-related spectral perturbation (ERSP) is a complementary measure, which estimates power at a given frequency in the signal.

\section{Results}

\section{Frequency discrimination thresholds}

Mean frequency discrimination limens (in natural log units) and the results from a two-way ANOVA (age band $\times$ SLI status) are shown in Table 2, indicating a main effect of age band (teenagers have lower thresholds than children) and an interaction between age band and SLI status. Contrasts conducted using $t$ tests indicated that the SLI-child and TD-child groups did not differ significantly $(t=0.87, \mathrm{df}=30, p=0.389)$, whereas the SLI-teen group did worse than the TD-teen group $(t=2.9, \mathrm{df}=28, p=$ $0.007)$. The Hertz equivalents of the thresholds are also shown in Table 2 and indicate that the mean thresholds for the TD-child group was just $>30 \mathrm{~Hz}$, the frequency difference used for the smaller deviant in the MMN study.

\section{Electrophysiological responses to standards and deviants}

The number of deviant trials included per condition did not differ by SLI status, but teenagers had slightly more included trials: means were $83.6(\mathrm{SD}=9.90)$ for TD-teen, $89.2(\mathrm{SD}=7.78)$ for TD-teen, 86.9 (SD = 9.46) for SLI-child, and 92.1 (SD = 7.27) for SLI-teen. All participants had at least 65 deviant trials per condition (for ANOVA, see supplemental Table S1, available at www. jneurosci.org as supplemental material).

To simplify data analysis, we followed the procedure of Bishop et al. (2010) and used the first spatial principal component to represent the auditory ERP. This avoided the need for analyzing
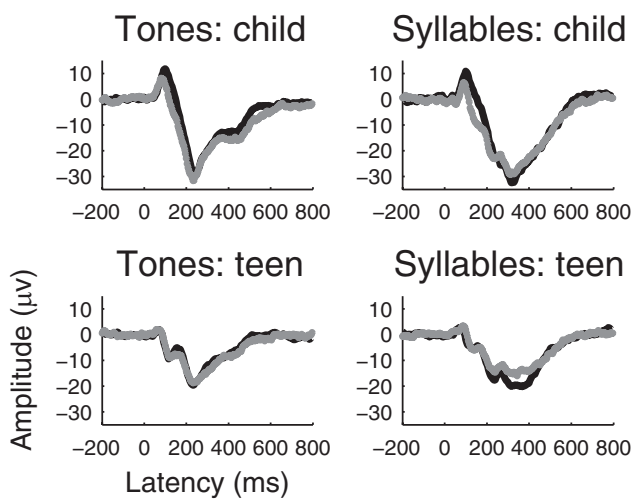

Figure 1. Average amplitude at principal component by age group and stimulus for TD (black line) and SLI (gray line). The N1 response is missing in the TD-child and SLI-child groups, whereas teenagers show a developing N1-P2 complex.

multiple electrodes while ensuring that all sites contributing to the auditory ERP were represented. The mean weights from each channel contributing to the principal component for each stimulus type and group are shown in head plots in supplemental Figure S1 (available at www.jneurosci.org as supplemental material), and indicate a similar frontocentral distribution in all four groups. Note that amplitudes of responses measured this way are substantially larger than those seen when a single electrode is considered.

Figure 1 shows the responses to standards and deviants based on the spatial principal component. Consistent with previous literature, there are striking differences between the waveforms for the children and teenagers. The N1 response is missing in the TD-child and SLI-child groups, as is usually found for children when stimuli are presented with stimulus onset asynchrony of $<1.4$ s (Ceponiené et al., 1998). The teenagers show a more adultlike pattern, with smaller amplitudes overall, and a developing N1-P2 complex. Although there are some differences in the amplitude of waveforms for TD versus SLI groups, the overall shape is similar at each age band.

Figure 2 shows the difference waveforms for each group. Dummy waveforms, created by subtracting the average for standards from each standard-before-deviant, are shown in addition to mismatch waveforms for deviants; these give an indication of how much amplitude variation is to be expected by chance. To identify regions where the averaged difference waveform for all participants fell significantly below zero, one-sample $t$ tests were conducted at each time-point. $t$ values $<-1.96$ are shown below the waveforms in Figure 2. As noted by Guthrie and Buchwald (1991), significance levels of $t$ values are misleading with this approach, because time points are not independent of one another, and a difference is only reliable when a sequence of $t$ values is significant, with the length of sequence depending on the autocorrelation of the waveform. In our study, the likelihood of obtaining spurious differences could be directly estimated by considering the dummy difference waveforms, where the same stimulus acts as both standard and deviant.

Following Bishop et al. (2010), we estimated the size of mismatch response by taking mean amplitude of the difference waveform in two time windows, 100-250 ms for the MMN and 350-550 ms for the LDN. For each time window, repeatedmeasures ANOVA was used with two within-subject factors, stimulus type (tones or speech) and deviant size (large or small), and two between-subject factors, SLI status (two levels) and age band (two levels). For the MMN, there were no main effects of the 


\section{Tones}

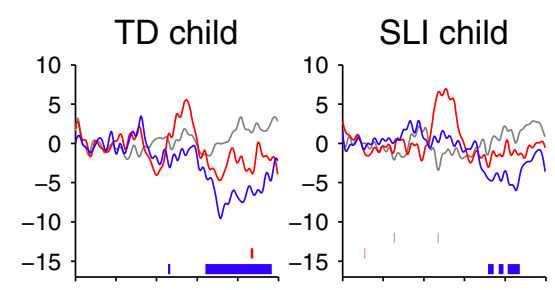

$-2000200400600800-2000200400600800$ TD teen
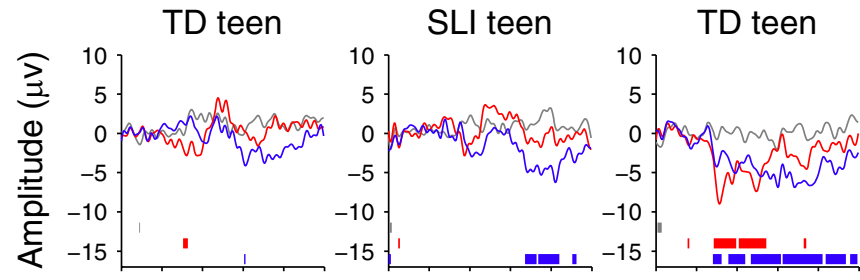

Syllables

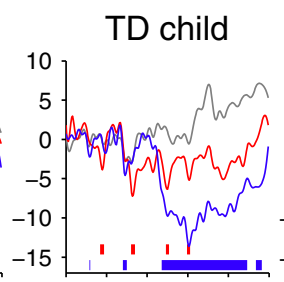

$-200 \quad 0200400600800$ Latency (ms)

Figure 2. Average mismatch responses from principal component by stimulus type and group for dummy difference files (gray), large deviants (red), and small deviants (blue). Regions where $t$ test value is $<-1.96$ are shown as bars of below the plot. In the MMN interval (100-250 ms postonset), the mismatch to speech stimuli is significantly greater than to tones, and mismatch to large deviants is greater than to small deviants, but group and age have no effect. For the LDN interval ( $350-550 \mathrm{~ms}$ ) mismatch is larger for the smaller deviant; this effect is significantly greater for TD than SLI participants.

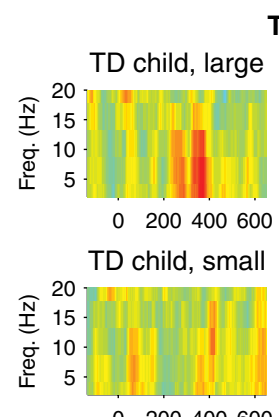

Tones
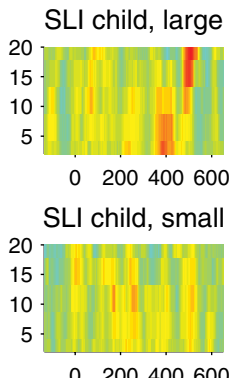

0200400600

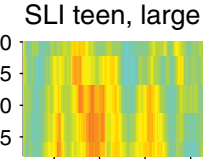

0200400600

0200400600

TD teen, small
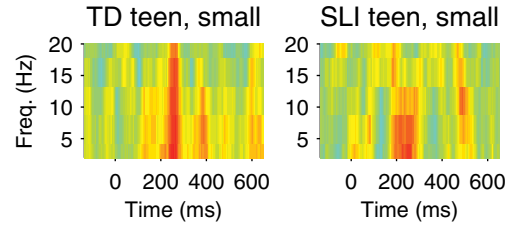

Figure 3. ITC for difference waveforms in relation to stimulus and group, with colors indicating range from zero, i.e., no synchronization (green), to 0.2 (deep red). The MMN region is characterized by a burst of increased ITC $<10 \mathrm{~Hz}$, which is similar for TD and SLI groups.

between-subjects factors of age and SLI status and no significant interactions with these factors. Main effects of stimulus type and deviant were significant, with MMN to speech stimuli being substantially larger than to tones $\left(F_{(1,60)}=7.75, p=0.007, \eta^{2}=\right.$ $0.114)$ and MMN to large deviants being greater than to small deviants $\left(F_{(1,60)}=5.34, p=0.024, \eta^{2}=0.082\right)$. Full ANOVA output is shown in supplemental Table S2 (available at www. jneurosci.org as supplemental material). ANOVA on peak latency showed no main effects or interactions, other than a main

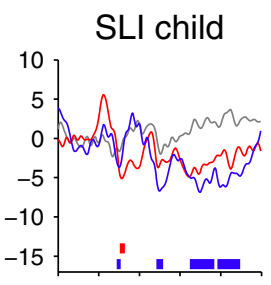

effect of stimulus type, and is shown in supplemental Table S3 (available at www. jneurosci.org as supplemental material).

A parallel analysis was conducted for the LDN interval, again showing significant main effects of stimulus type and deviant size, but this time the latter effect reflected a larger LDN to the smaller deviant, an effect previously reported by Bishop et al. (2010), who included a subset of the current TD sample in their analysis. Neither age band nor SLI status gave a significant main effect (for full output, see supplemental Table S4, available at www.jneurosci.org as supplemental material), but there was a significant interaction between SLI status and deviant size $\left(F_{(1,60)}=4.48, p=0.038, \eta^{2}=0.070\right)$. This was followed up using $t$ tests. The mean LDN to the large deviant was -1.63 $(\mathrm{SD}=5.50)$ for $\mathrm{TD}$ participants and $-1.43(\mathrm{SD}=5.17)$ for SLI participants, a nonsignificant difference $\left(t_{(62)}=0.15\right.$, $p=0.878)$. For the small deviants, the mean LDN for TD participants was -5.69 $(\mathrm{SD}=4.36)$, whereas that for SLI participants was $-2.37(\mathrm{SD}=5.44)$, a significant difference $\left(t_{(62)}=2.69, p=0.009\right)$. Thus, the analysis confirmed the impression seen in Figure 2-there is a greater LDN for TD than for SLI participants for the small deviant only.

\section{Time-frequency analysis}

Figure 3 shows the mean levels of ITC for mismatch waveforms in frequencies ranging from 1 to $20 \mathrm{~Hz}$ for the four groups. This indicates the extent to which individual mismatch waveforms are synchronized in phase at different frequencies. In all groups, one can see the phenomenon described by Bishop et al. (2010) whereby the MMN region is characterized by a burst of increased ITC that is particularly marked in frequencies $<10 \mathrm{~Hz}$. Visual inspection does not, however, suggest any substantial differences between SLI and TD participants; this was confirmed by ANOVA on the mean ITC value in the theta frequency band, which found no main effect or interaction for SLI status (supplemental Table S5, available at www. jneurosci.org as supplemental material).

Figure 4 shows corresponding data for the ERSP, which measures the amount of power in the difference waveform at different time points, relative to power in the baseline. A striking and hitherto unremarked phenomenon can be seen in the TD plots, namely a decrease in power across a broad frequency band in the later half of the trial. This was quantified by taking mean ERSP in the theta band $(4-7 \mathrm{~Hz})$ for the second half of the trial, from 300 to $600 \mathrm{~ms}$. ANOVA revealed that this index gave a significant main effect of SLI status $\left(F_{(1,60)}=9.67, p=\right.$ $\left.0.003, \eta^{2}=0.139\right)$, whereas the effect of age was close to zero and 
nonsignificant (for full ANOVA, see supplemental Table S6, available at www. jneurosci.org as supplemental material). The only other significant effects were a two-way interaction between SLI status and deviant size, and a three-way interaction between SLI status, age band, and deviant size. This latter interaction reflects the phenomenon apparent in Figure 4, whereby a small deviant is associated with a large decrease in power in TD participants, but an increase in power in SLI participants, with the effect especially prominent in older children. An ANOVA was run to check whether this might arise from a difference in baseline power (supplemental Table S6, available at www. jneurosci.org as supplemental material); this revealed a large effect of age, but no main effect of language status, and no interaction of language status with age, stimulus type, or deviant. Supplemental Figure S2 (available at www.jneurosci.org as supplemental material) shows the mean log power in different frequency bands without baseline correction.

\section{Relationship between indices of mismatch responses and behavioral measures}

We inspected correlations between mismatch indices and both frequency discrimination and nonword repetition, with the expectation that mismatch responses to tones might be correlated with frequency discrimination, and those to speech would correlate with nonword repetition. To reduce the number of correlations considered, focus was restricted to the small deviants, giving 16 correlations and a Bonferroni-adjusted $p$ value of 0.003 . As can be seen in Table 3, we did not replicate the finding by Bishop et al. (2010) of a correlation between frequency discrimination (FD) and early ITC, and none of the other mismatch indices for tones related to FD threshold. However, there was a significant negative correlation between the ERSP to syllables in the later time interval and nonword repetition score $(r=-0.4, N=61, p=0.001)$. This indicates that participants who are poor at nonword repetition fail to show a drop in power corresponding to event-related desynchronization. This correlation was recomputed separately for TD and SLI groups to see whether it was driven solely by the TD/SLI group differences. Within the TD group, it was nonsignificant $(r=-0.056, N=29)$, but it remained significant within the SLI group $(r=-0.395, N=32, p=0.032)$ (for the scatterplot, see supplemental Fig. S3, available at www.jneurosci.org as supplemental material).

\section{Discussion}

\section{Overview of findings on SLI}

Data from this study help resolve some of the inconsistent findings in this field. Previous studies have disagreed as to whether children with SLI have reduced mismatch responses to auditory signals, and if so, whether they are confined to speech sounds (Bishop, 2007). We found abnormal responses in SLI, but only for the LDN time range, not the earlier MMN. Some researchers have blurred the distinction between these responses, labeling them as early and late MMN. However, they appear to reflect

\section{Tones}

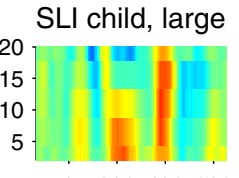

0200400600
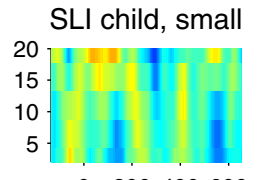

0200400600

SLI teen, large

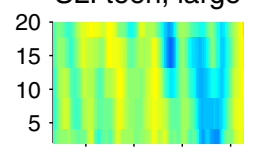

0200400600

SLI teen, small

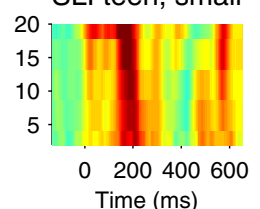

Syllables

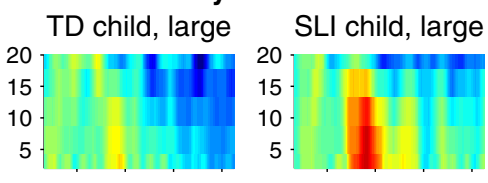

0200400600

0200400600

TD child, small

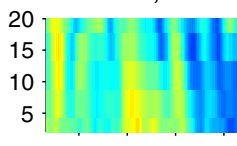

0200400600

TD teen, large

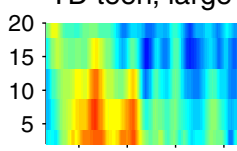

0200400600

TD teen, small
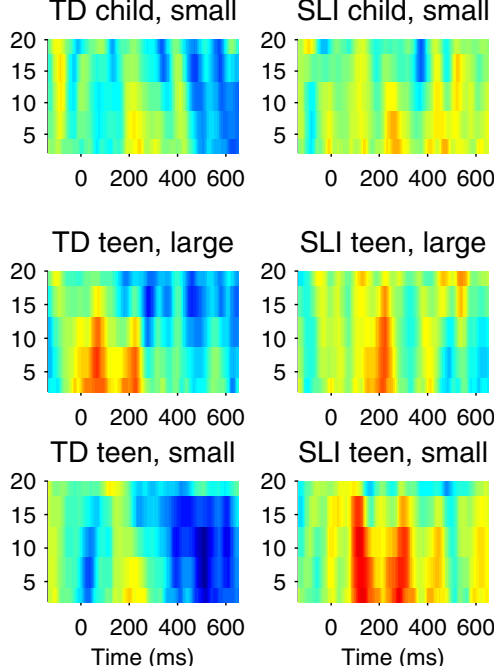

0200400600

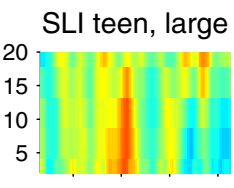

0200400600
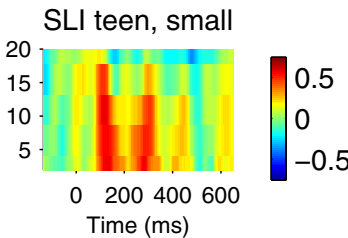

\begin{tabular}{lcc}
\hline Small deviant mismatch indices & $\begin{array}{c}\text { Frequency discrimination } \\
\text { skill }(N=62)\end{array}$ & $\begin{array}{c}\text { Nonword repetition } \\
(N=61)\end{array}$ \\
\hline Tones MMN mean amplitude & 0.024 & 0.046 \\
Tones LDN mean amplitude & -0.044 & -0.018 \\
Tones ITC 0 -300 ms, 4-7 Hz & 0.114 & 0 \\
Tones ERSP 300 - 600 ms, 4-7 Hz & 0.099 & -0.217 \\
Syllables MMN mean amplitude & -0.218 & 0.007 \\
Syllables LDN mean amplitude & 0.243 & -0.094 \\
Syllables ITC $0-300 \mathrm{~ms}, 4-7 \mathrm{~Hz}$ & 0.010 & 0.113 \\
Syllables ERSP 300 - 600 ms, 4-7 Hz & -0.138 & -0.400
\end{tabular}

Correlation of 0.37 or more is significant at Bonferroni-corrected level of $0.05 / 16$.

${ }^{a}$ Thresholds inverted so that a positive correlation means good skill corresponding to positive mismatch index.

sequential stages in processing an auditory signal, show different developmental courses, and show opposite effects of size of difference between standard and deviant. One might wonder whether the lack of group differences on the MMN reflects the fact that this component is much smaller in children than adults, when quantified as mean amplitude in the time window 100-250 ms. However, no group difference is seen when the MMN is assessed using ITC, a measure of phase coherence across trials. This gives a more sensitive measure of MMN in children than averaged mean amplitude because it is unaffected by the polarity of a response and so will reflect positive as well as negative mismatch responses. As can be seen in Figure 2, a positive mismatch response is evident after an initial small negativity and the ITC is significant during this period. This effect, however, is at least as strong in those with SLI as in the TD group. We therefore conclude that the initial brain response indicating detection of mismatch between memory of the standard and an incoming auditory signal is intact in SLI for both speech and nonspeech signals. This suggests that the perceptual anchor theory does not 
apply to SLI; this theory, which was proposed for dyslexia, maintains there is difficulty in forming a long-term representation of a repeating standard in memory (Ahissar et al., 2006). A difference between SLI and TD groups is, however, apparent in the later interval corresponding to the LDN. We discuss the significance of this finding further below.

The lack of significant correlations between indices of mismatch responses to small frequency deviants and FD thresholds in this study is puzzling in view of the significant association between early-interval ITC and FD thresholds found by Bishop et al. (2010) in a larger normative sample that covered the whole age range from children to adults and included a subset of the current TD sample. There are two possibilities. First, the finding of raised FD thresholds in the SLI-teen group may have different causes from the abnormal LDN to the same stimuli seen in SLI individuals of all ages. For instance, the behavioral task has attentional and memory demands, which may be important. The second, more prosaic explanation is in terms of the low reliability of electrophysiological indices of mismatch responses in individuals. Bishop and Hardiman (2010) demonstrated good reliability of mismatch indices in adults when 300 deviant responses were available, but this declined when a smaller number of trials was used. We had $\sim 85-90$ trials per condition on average in this study, and it is likely that this may be sufficient to demonstrate clear effects at the group level, but too few for reliable estimates in individuals. The current study did, however, reveal one significant correlation between behavior and electrophysiological response, namely that the negative correlation between ERSP to small speech deviants in the later part of the epoch and performance on a nonword repetition task. This observation merits further investigation, given that impaired nonword repetition is one of the most reliable markers of heritable forms of SLI (Bishop et al., 1996).

\section{Implications for our understanding of the nature of mismatch responses in the auditory ERP}

Time-frequency analysis in this study provided new insights into the mechanisms underlying generation of the MMN and LDN, emphasizing the difference between these mismatch responses. MMN appears to arise from an enhancement of phase synchronization in the theta frequency band (Bishop and Hardiman, 2010; Bishop et al., 2010). In typically developing children, we found that LDN is accompanied by a reduction of power in a relatively wide frequency band encompassing delta, theta, and low alpha. Pfurtscheller and Lopes da Silva (1999) termed a decrease in power in a specific frequency band as event-related desynchronization (ERD) and proposed it reflected a reduction in synchrony of activity in an underlying neuronal population. Most studies of ERD involved memory or motor tasks and focused on alpha or beta frequency bands. An exception is a study by Edwards et al. (2009), who measured electrophysiological responses from the cortical surface to auditory speech stimuli in nine adult patients who were being evaluated before neurosurgery. They found a reduction of power at $7-14 \mathrm{~Hz}$ relative to baseline (ERD) in the range $200-400 \mathrm{~ms}$ postonset, which was correlated with a prior enhancement of power in the gamma band at 50-200 ms postonset. Although they used an oddball paradigm, their analysis focused on responses to standard and deviant stimuli, rather than difference waveforms, and so is not directly comparable to our study, but it suggests that the lowerfrequency ERD that we observe in the later portion of the difference waveforms is an exaggeration of the normal brain response to auditory stimuli.
The physiological basis of ERD is still poorly understood, but Pfurtscheller and Lopes da Silva (1999) noted that it is influenced by interconnectivity in neuronal circuits. One aspect of neural maturation that develops throughout childhood is greater specificity in synaptic connectivity as nonfunctional connections are pruned. This underlies the development of more focal representations of functions in the brain. High spectral power is seen when there is a large number of coherently activated neurons; thus, the ERSP profile of typical children could indicate a transition from an early stage of detection of a deviant stimulus by a large brain region (indexed by an assembly of neurons responding in phase to give high ITC) to a later stage of stimulus identification requiring more focal brain activity, indexed by a decrease in ERSP below baseline levels. According to this model, the fact that ERD is reduced or absent in children with SLI could indicate that they have less specialized brain maps for differentiation of auditory stimuli. Identification of a change from standard to deviant would lead to altered oscillatory activity in a smaller region of cortex in TD children than in those with SLI, and hence correspond to more event-related desynchronization as activity becomes more focal. This highly speculative account has features in common with Tallal's (2004) proposals about coarse neural coding of auditory stimuli in SLI, but it is more general, in that it is not restricted to brief or rapid stimuli. Tallal's theory has been extended to account for developmental dyslexia as well as SLI, and it will be of considerable interest to see whether time-frequency analysis can reveal similar patterns of response in children who have milder difficulties that predominantly affect written language.

\section{Implications for a maturational lag account of SLI}

Hautus et al. (2003), McArthur and Bishop (2004), and Wright and Zecker (2004) proposed that that auditory perceptual difficulties seen in some children with developmental speech-language disorders may correspond to a maturational lag rather than a static deficit. For instance, if there were little change in ability on a task from age 5 to 10 years followed by an improvement in adolescence, then a 10 -year-old with a 4 -year lag in performance might appear unimpaired in the preteen years, but a deficit would become apparent in adolescence. The pattern of results on the behavioral FD task obtained here fits that picture, and if we had only behavioral data to rely on, the maturational lag account would seem the best explanation. The electrophysiological data, however, tell a different story. There are clear developmental changes in mismatch responses (Bishop et al., 2010), but children with SLI do not appear to be immature. Rather, they show atypical mismatch responses, regardless of age, the most striking feature of which is a lack of the usual power reduction at lower frequencies in the second half of the trial.

In conclusion, the current study illustrates the value of analyzing oscillatory aspects of ERP data alongside more traditional averaging approaches. Key oscillatory changes are often invisible in the averaged signal, yet can be uncovered by single trial analysis. There is growing interest in using this approach to study neurophysiological maturation (Shahin et al., 2010). Such an approach is feasible for analyzing mismatch responses, where single trial difference waveforms can be created by subtraction of the averaged standard response from each trial, and, as we have shown, has promise for resolving some long-standing debates about the underlying causes of neurodevelopmental disorders and throwing light on the biological bases of language impairments. 


\section{References}

Ahissar M, Lubin Y, Putter-Katz H, Banai K (2006) Dyslexia and the failure to form a perceptual anchor. Nat Neurosci 9:1558-1564.

Bailey PJ, Snowling MJ (2002) Auditory processing and the development of language and literacy. Brit Med Bull 63:135-146.

Bishop DV (2003a) The Children's Communication Checklist, version 2 (CCC-2). London: Pearson.

Bishop DV (2003b) The Test for Reception of Grammar, version 2 (TROG2). London: Pearson.

Bishop DV (2004) Expression, Reception and Recall of Narrative Instrument (ERRNI). London: Psychological Corporation.

Bishop DV (2007) Using mismatch negativity to study central auditory processing in developmental language and literacy impairments: where are we, and where should we be going? Psychol Bull 133:651-672.

Bishop DV, Hardiman MJ (2010) Measurement of mismatch negativity in individuals: a study using single-trial analysis. Psychophysiology 47:697-705.

Bishop DV, Snowling MJ (2004) Developmental dyslexia and specific language impairment: same or different? Psychol Bull 130:858-886.

Bishop DV, North T, Donlan C (1996) Nonword repetition as a behavioural marker for inherited language impairment: evidence from a twin study. J Child Psychol Psychiatry 37:391-403.

Bishop DV, Hardiman MJ, Barry JG (2010) Is auditory discrimination mature by middle childhood? A study using time-frequency analysis of mismatch responses from 7 years to adulthood. Dev Sci. Advance online publication. doi:10.1111/j.1467-7687.2010.00990.x.

Boersma P, Weenink D (2009) Praat: a system for doing phonetics by computer. Glot Internat 5:341-345.

Ceponiené R, Cheour M, Näätänen R (1998) Interstimulus interval and auditory event-related potentials in children: evidence for multiple generators. Electroencephalogr Clin Neurophysiol 108:345-354.

Cheour M, Leppänen PH, Kraus N (2000) Mismatch negativity (MMN) as a tool for investigating auditory discrimination and sensory memory in infants and children. Clin Neurophysiol 111:4-16.

Cheour M, Korpilahti P, Martynova O, Lang AH (2001) Mismatch negativity and late discriminative negativity in investigating speech perception and learning in children and infants. Audiol Neurootol 6:2-11.
Craig C (2008) GoldWave (Version 5.23)sound editing program. St Johns: GoldWave.

Delorme A, Makeig S (2004) EEGLAB: an open source toolbox for analysis of single-trial EEG dynamics including independent component analysis (sccn.ucsd.edu/eeglab/). J Neurosci Methods 134:9-21.

Eaves LC, Wingert HD, Ho HH, Mickelson EC (2006) Screening for autism spectrum disorders with the social communication questionnaire. J Dev Behav Pediatr 27(2 Suppl):S95-S103.

Edwards E, Soltani M, Kim W, Dalal SS, Nagarajan SS, Berger MS, Knight RT (2009) Comparison of time-frequency responses and the event-related potential to auditory speech stimuli in human cortex. J Neurophysiol 102:377-386.

Guthrie D, Buchwald JS (1991) Significance testing of difference potentials. Psychophysiology 28:240-244.

Hautus MJ, Setchell GJ, Waldie KE, Kirk IJ (2003) Age-related improvements in auditory temporal resolution in reading-impaired children. Dyslexia 9:37-45.

Korkman M, Kirk U, Kemp SI (1998) NEPSY: a developmental neuropsychological assessment. San Antonio: Psychological Corporation.

McArthur GM, Bishop DV (2004) Which people with specific language impairment have auditory processing deficits? Cogn Neuropsychol 21:79-94.

Pfurtscheller G, Lopes da Silva FH (1999) Event-related EEG/MEG synchronization and desynchronization: basic principles. Clin Neurophysiol 110:1842-1857.

Rutter M, Bailey A, Lord C (2003) Social Communication Questionnaire (SCQ). Los Angeles: Western Psychological Services.

Shahin AJ, Trainor LJ, Roberts LE, Backer KC, Miller LM (2010) Development of auditory phase-locked activity for music sounds. J Neurophysiol 103:218-229.

Tallal P (2004) Improving language and literacy is a matter of time. Nat Rev Neurosci 5:721-728.

Torgesen JK, Wagner R, Rashotte C (1999) Test of Word Reading Efficiency (TOWRE). New York: Psychological Corporation.

Wechsler D (1999) Wechsler Abbreviated Scale of Intelligence (WASI). San Antonio: Psychological Corporation.

Wright BA, Zecker SG (2004) Learning problems, delayed development, and puberty. Proc Natl Acad Sci U S A 101:9942-9946. 